\title{
New Insights Into Fatty Liver Preservation Using Institute Georges Lopez
}

Preservation Solution

M.A. Zaoualí, I. Ben Mosbah, H. Ben Abdennebi, M. Calvo, E. Boncompagni, O. Boillot, C.

Peralta, and J. Roselló-Catafau

One of the major challenges is the increased use of "marginal" grafts for liver transplantation. This fact has obliged physicians to use liver grafts with moderate steatosis, although they show higher vulnerability against cold ischemiareperfusion injury than normal livers. Steatosis results from an abnormal accumulation of fat in hepatic sinusoids, leading to a severe obstruction of hepatic flow with important alterations in liver microcirculation.

The composition of the preservation solution is a crucial factor in the preservation of a liver graft, especially when the liver is steatotic. Although the University of Wisconsin (UW) is useful for liver graft preservation, its limitations are well known; UW contains high $\mathrm{K}_{-}(125 \mathrm{mmol} / \mathrm{L})$ and low $\mathrm{Na}_{-}(30 \mathrm{mmol} / \mathrm{L})$ concentrations, which provoke vasoconstriction. In addition, the presence of the colloid, hydroxylethyl statch (HES), can trigger red blood cell aggregation, thereby promoting stasis in hepatic sinusoids and limiting the efficiency of graft washout, thereby exacerbating microcirculatory disturbances in steatotic livers and compromising graft outcomes after transplantation.

Recently, we have described Institute Georges Lopez preservation solution (IGL-1) as an effective alternative to UW. IGL-1 composition is characterized by the reversion of the ionic $\mathrm{K} / \mathrm{Na}$ concentrations and the substitution of $\mathrm{HES}$ by polyethylene glycol 35 (PEG-35). These characteristics of IGL-1 render it suitable for abdominal organ preservation including fatty liver grafts. The mechanisms induced by the use of IGL-1 solution to prevent liver cold iscemia-reperfusion injury include influences on cell signaling in fatty livers.

IGL-1 solution effectively protects normal and moderately steatotic livers against cold ischemia-resperfusion injury. These beneficial effects have initially been associated with the involvement of freely diffusible molecules, such as nitric oxide (NO), which act in an autocrine and/or paracrine manner to effectively trigger and differentially activate cytoprotective cell signaling pathways during cold ischemiareperfusion, namely, hypoxia-induced factor-1 (HIF-1) and heme oxygenase-1 (HO-1), respectively.

The benefits of IGL-1 are mediated by NO. Constitutive nitric oxide synthase is activated, leading to the generation of $\mathrm{NO}$, which efficiently protects fatty livers against cold ischemia-reperfusion. This change was evidenced by prevention of steatotic and nonsteatotic liver injuries (diminished aspartate transferase/alanine transferase), as well as an enhanced hepatic function as evidenced by increased bile production and diminished vascular resistance. The advantages of IGL-1 were exacerbated when an anti-ischemia drug such as trimetazidine (antioxidant and NO donor) was used as an additive. 
NO is a diffusible, vasodilator molecule generated by liver endothelia. It has a pivotal, dual role: it prevents exacerbated oxidative stress from occurring in fatty livers subjected to ischemia-reperfusion injury and liver transplantation, and it ameliorates the deficient microcirculation existing in steatotic livers, as a consequence of fat accumulation in hepatic sinusoids, contributing thus to improve the hepatic tissue oxygenation. Additional benefits of NO generation in preserved livers may be associated with its inhibitory action on endothelins, and its potent effects to counter liver vasoconstrictor peptides formed during liver graft reperfusion.

In addition, most current data have focused on whether NO regulates cytoprotective signal transduction pathways during cold storage and normothermic reperfusion, as HIF-1 and $\mathrm{HO}-1$, which seem to ameliorate fatty liver preservation.

Lack of an oxygen supply to the graft induces activation of HIF-1, as an "adaptative response" to oxygenation changes occurring during cold preservation. Active HIF-1 is a heterodimer consisting of an inducible HIF-1 alpha subunit and another constitutively expressed molecule called HIF-1 beta. During ischemia/hypoxia, HIF1 alpha accumulates but is extremely labile in normoxic conditions. Its stability is drastically reduced by the oxygen-dependent enzymatic hydroxylation of proline residues by prolyl hydroxylase enzymes. It has been shown that NO impairs normoxic degradation of HIF-1 alpha by inhibition of HIF-1 prolyl-hydroxylases. This fact contributes to stabilization and subsequent accumulation as previously reported by several authors.

Significant activation of HIF-1 alpha originates in fatty livers preserved in IGL-1 solution. In addition, HIF-1 alpha is maintained after normoxic reperfusion as a consequence of $\mathrm{NO}$ generation occurring in IGL-1 cold preservation. It has been reported by several authors that NO stabilizes HIF-1 alpha in normoxia by directly inhibiting prolyl-hydroxylase enzymes.

Importantly, HIF-1 signaling has been implicated in the development of hypoxiainduced delayed protection. In this line, recent studies have shown that cytoprotective effects of HIF-1 are closely related to HO-1.It is clear that HO-1 upregulation protects a fatty liver from a genetically fat Zucker rat liver against ischemia-reperfusion injury20, consequently, it improves extended preservation of liver grafts. HIF-1 accumulation among fatty livers preserved in IGL-1 occurred concomitant with overexpression of $\mathrm{HO}-1$ after reperfusion favoring graft protection against cold ischemia-reperfusion injury.

In summary, NO is generated during IGL-1 static preservation and is crucial for enhanced preservation of fatty liver grafts. It up-regulates important protective cell signaling as HIF-1 alpha and $\mathrm{HO}-1$, which are important for improving liver graft recovery, especially when steatosis is present. 